\begin{tabular}{|c|c|}
\hline Title & A ccelerated FDTD A naly sis of A ntennas L oaded by Electric Circuits \\
\hline Author(s) & W atanabe, Y uta; Igarashi, Hajime \\
\hline Citation & $\begin{array}{l}\text { IEEE Transactions on A ntennas and Propagation, 60(2), } 958-963 \\
\text { https://doi.org/10.1109/T AP.2011.2173148 }\end{array}$ \\
\hline Issue Date & 2012-02 \\
\hline Doc URL & http:/hdl.handle.net/2115/49503 \\
\hline Rights & $\begin{array}{l}\text { (c) } 2012 \text { IEEE. Reprinted, with permission, from Y uta W atanabe; Hajime Igarashi, A ccelerated FDTD A nalysis of } \\
\text { A ntennas L oaded by Electric Circuits, IEEE Transactions on A ntennas and Propagation, Feb. 2012. This material is } \\
\text { posted here with permission of the IEEE. Such permission of the IEEE does not in any way imply IEEE endorsement of } \\
\text { any of Hokkaido University products or services. Internal or personal use of this material is permitted. However, } \\
\text { permission to reprint/republish this material for advertising or promotional purposes or for creating new collective } \\
\text { works for resale or redistribution must be obtai ned from the IEEE by writing to pubs permissions@ ieee.org. By } \\
\text { choosing to view this document, you agree to all provisions of the copyright laws protecting it. }\end{array}$ \\
\hline Tyре & article (author version) \\
\hline File Information & ToAP60-2_958-963.pdf \\
\hline
\end{tabular}

Instructions for use 


\title{
Accelerated FDTD Analysis of Antennas Loaded by Electric Circuits
}

\author{
Yuta Watanabe, Student member, IEEE and Hajime Igarashi, Member, IEEE
}

\begin{abstract}
A fast FDTD method for the analysis of antennas loaded by non-linear electric circuits is introduced. In the present analysis, the modified nodal analysis (MNA) method is coupled with the FDTD method. The time-periodic explicit error correction (TP-EEC) method is applied to the MNA method for accelerated computation of the transient processes. The present method is applied to analysis of simplified models of an RFID tag composed of a non-linear electric circuit and line antenna. It is shown that the present method can effectively shorten the computational time by accelerating the transient processes.
\end{abstract}

Index Terms-FDTD method, TP-EEC method, Electromagnetic waves, Modified nodal analysis, RFID tag

\section{INTRODUCTION}

$\mathrm{F}$ IELD COMPUTATION METHODS, such as the finite -difference time-domain (FDTD) method [1], [2] and the method of moment [3], have widely been used for analysis of high frequency electronic devices. In recent years, these methods have been applied to coupling analysis of high-frequency electromagnetic fields and electric circuits for the design of high-frequency electronic devices and analysis of electromagnetic compatibility (EMC) problems [4]. In the coupling analysis, circuit simulation involving nonlinearity requires time domain computations. For this reason, the coupling analysis of electromagnetic fields and nonlinear electric circuits usually requires high computational cost. When the time constant of the circuit is much longer than the time period of electromagnetic waves, this problem becomes quite severe because the number of time steps must be considerably large. It would be possible to reduce the computational cost if one could effectively shorten the time constant, that is, accelerate the computation of the transient processes of the circuit.

The time-periodic explicit error correction (TP-EEC) method [5], [6], which accelerates the transient processes of time-periodic systems, has been introduced for reduction of computational costs. The TP-EEC method is based on the assumption that the unknown variables are temporally periodic in the steady state and slowly converging components without periodicity can be separated from them. The slowly converging components are then determined by solving small-scale correction equations to correct the transient solutions. The TP-EEC method is an extension of the EEC method [7], which gives a general framework of the error correction based on the decomposition of unknowns to fast and slowly converging components. It has been shown that the EEC has a common theoretical basis with the deflation methods [8], which have been applied to linear systems of poor convergence [9], [10]. The TP-EEC method has been applied to finite element (FE) analysis of motors and coupling FE analysis of circuit and eddy current fields [6], in the latter of which inductance is computed in the FE analysis by taking magnetic saturation into account.

In this paper, we will discuss the effectiveness of the TP-EEC method when applied to antenna analysis, where coupling between electromagnetic waves and a non-linear circuit is considered. In particular, we consider here transient analysis of dipole antennas loaded by a non-linear circuit, which are simplified models of the UHF-band RFID tag. In the design optimization of antennas for RFID tags, the coupled problem between the electromagnetic waves and the circuit must be repeatedly solved [11]. Hence the reduction in the computational cost for the coupling analysis is of fundamental importance. Moreover, in this paper, we will introduce a theoretical basis of the TP-EEC method for explanation of the reason why it is effective for acceleration of the transient processes.

In this work, the FDTD method and Modified Nodal Analysis (MNA) [12], [13] are employed for the coupling analysis of a high frequency electromagnetic field and a non-linear circuit. This paper will be organized as follows: in Section II, the coupled method with the FDTD method and MNA will be formulated. Moreover a computational procedure of the present method will be described. In Section III, the TP-EEC method will be formulated, and effect of the TP-EEC method will be discussed, while in Section IV, numerical results will be shown to verify the present method.
Manuscript received October 9, 2001.

Y. Watanabe and H. Igarashi are with the Graduate School of Information Science and Technology, Hokkaido University, Sapporo, 060-0814, Japan (phone: +81 11-706-6488; fax: +81 706-7670; e-mail: ywata@ em-si.eng.hokudai.ac.jp). 


\section{HYBRIDIZATION OF FDTD METHOD AND MNA}

The hybridization of the FDTD method and MNA will be described in the following. The Maxwell equations

$\varepsilon \frac{\partial \boldsymbol{E}}{\partial t}+\boldsymbol{J}(\boldsymbol{E})=\nabla \times \boldsymbol{H}$

$\mu \frac{\partial \boldsymbol{H}}{\partial t}=\nabla \times \boldsymbol{E}$

are considered in this paper where the conduction current density $\boldsymbol{J}$ is determined from the voltage-current characteristics of the non-linear circuit. In the FDTD process, (1-a) and (1-b) are approximated by the central differences in time and space and explicitly solved in turns as follows [2]:

$$
\begin{aligned}
& \boldsymbol{E}^{n}=\frac{1-\sigma \Delta t / 2 \varepsilon}{1+\sigma \Delta t / 2 \varepsilon} \boldsymbol{E}^{n-1}+\frac{\Delta t / \varepsilon}{1+\sigma \Delta t / 2 \varepsilon} \nabla \times \boldsymbol{H}^{n-1 / 2} . \\
& \boldsymbol{H}^{n+1 / 2}=\boldsymbol{H}^{n-1 / 2}+\frac{\Delta t}{\mu} \nabla \times \boldsymbol{E}^{n} .
\end{aligned}
$$

Let us consider the line antenna loaded by a non-linear circuit, shown in Fig. 1, parallel to the $\mathrm{z}$-axis, where $\Delta X, \Delta Y$ and $\Delta Z$ are the cell size of the FDTD method. The spatial size of the non-linear circuit is assumed to be sufficiently smaller than that of the FDTD cell. By integrating (1-a) on the surface $S$ of the FDTD cell, we obtain

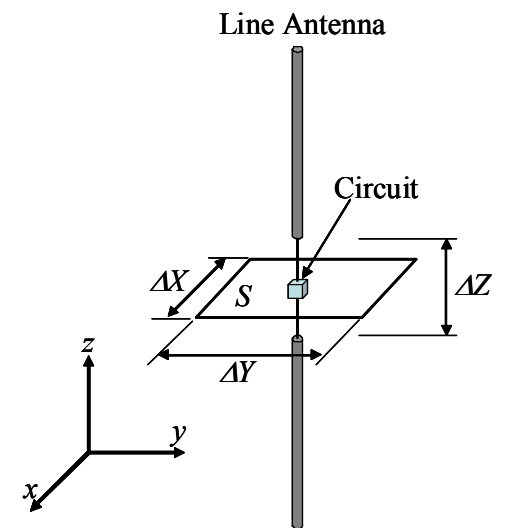

Fig. 1. Line antenna loaded by non-linear circuit.

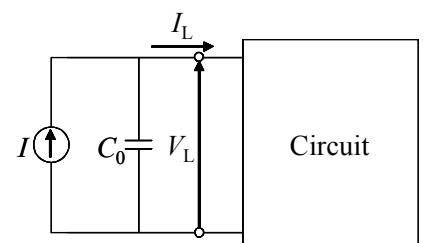

Fig. 2. Equivalent circuit for FDTD method of antenna loaded by non-linear circuit.

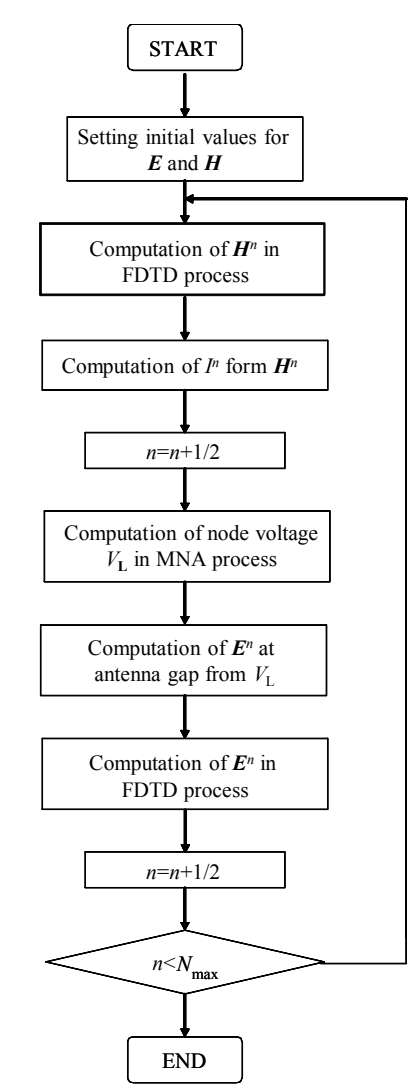

Fig. 3. Flow diagram.

$C_{0} \frac{\partial V_{L}}{\partial t}+I_{L}\left(V_{L}\right)=I$

where $V_{\mathrm{L}}=E_{\mathrm{Z}} \Delta Z$ is the voltage imposed to the circuit, $C_{0}=\varepsilon \Delta X \Delta Y / \Delta Z$ is the capacitance of the FDTD cell, $I_{\mathrm{L}}$ is the current flowing into the non-linear circuit and the $I$ is total current given by

$I=\int_{\partial S} \boldsymbol{H} \cdot \mathrm{d} \boldsymbol{s}$.

The equivalent circuit governed by (3) is shown in Fig. 2 [12], [13]. This circuit is composed of a parallel circuit of the current source $I$ computed from (4), the capacitance $C_{0}$ and the non-linear circuit. The equivalent circuit shown in Fig. 2 is analyzed by MNA in this work. Modified nodal analysis determines the voltages between nodes in the electrical circuit according to Kirchhoff's Current Law. A system of the non-linear circuit equations of the form

$\boldsymbol{f}(\boldsymbol{x})+\mathrm{C} \frac{\mathrm{d} \boldsymbol{x}}{\mathrm{d} t}=\boldsymbol{b}(t)$

is solved, where $\boldsymbol{x}(t) \in \mathbb{R}^{N}$ is composed of the unknown nodal voltages, $\boldsymbol{b}(t)$ current source driven by the antenna, $\boldsymbol{f}$ non-linear function which includes the effects of the active devices such as diodes and transistors, and $\mathrm{C}$ is the capacitance matrix. Note 
here that equation (5) includes (3).

Fig. 3 shows the flow diagram of the coupling analysis of the FDTD method and MNA. In the FDTD process, the magnetic field $\boldsymbol{H}^{n-1 / 2}$ is computed, where $n$ denotes the time step, and $I^{n-1 / 2}$ is computed from $\boldsymbol{H}^{n-1 / 2}$ by (4). Then, $I^{n-1 / 2}$ is substituted to the right-hand side of (5), which is solved by MNA for $\boldsymbol{x}$ including $V_{L}{ }^{n}$. The resultant electric field $E_{\mathrm{z}}{ }^{n}=V_{L} / \Delta Z$ is substituted to (2-b), which is solved by the FDTD method for $\boldsymbol{H}^{n+1 / 2}$. These computations of $\boldsymbol{E}, \boldsymbol{H}, I$ and $V_{L}$ are repeated until a steady solution is obtained.

\section{ACCELERATION OF CONVERGENCE IN CIRCUIT ANALYSIS}

This section describes the TP-EEC method which accelerates the transient processes of the circuit.

\section{A. Discretized Circuit Equation}

Discretization of (5) with the finite difference leads to a system of nonlinear equations of the form

$\boldsymbol{C}\left(\boldsymbol{x}_{n}\right)+\boldsymbol{P}\left(\boldsymbol{x}_{n-1}\right)=\tilde{\boldsymbol{b}}$

where

$$
\begin{aligned}
& \boldsymbol{C}\left(\boldsymbol{x}_{n}\right)=\theta \boldsymbol{f}\left(\boldsymbol{x}_{n}\right)+\mathrm{C} \frac{\boldsymbol{x}_{n}}{\Delta t} \\
& \boldsymbol{P}\left(\boldsymbol{x}_{n}\right)=(1-\theta) \boldsymbol{f}\left(\boldsymbol{x}_{n}\right)-\mathrm{C} \frac{\boldsymbol{x}_{n}}{\Delta t} \\
& \tilde{\boldsymbol{b}}=\theta \boldsymbol{b}_{n}+(1-\theta) \boldsymbol{b}_{n-1}
\end{aligned}
$$

and $0 \leq \theta \leq 1$. When $\theta$ equals to 1 , for example, this scheme corresponds to backward Euler method. In this work, (6) is solved with the Newton-Raphson method at each time step.

\section{B. TP-EEC Method}

When the time constant of the system governed by (6) is much longer than $\Delta t$, high computational cost is required to obtain the steady state solutions which do not vary in time. To accelerate the convergence to the steady state, the TP-EEC method is applied to (6).

It is assumed that $\boldsymbol{b}_{n}$ is periodic, which satisfies $\boldsymbol{b}_{0}=\boldsymbol{b}_{N \mathrm{~s}}$. The solution $\boldsymbol{x}_{n}$ to (6) is then expected to have periodicity in the steady state. On the other hand, in the transient state, periodicity would approximately hold, that is,

$$
\boldsymbol{x}_{0} \approx \boldsymbol{x}_{N_{S}}
$$

To apply the TP-EEC method to (6), we introduce the vectors defined for each period as
$\boldsymbol{X}=\left[\begin{array}{c}\boldsymbol{x}_{1} \\ \boldsymbol{x}_{2} \\ \vdots \\ \boldsymbol{x}_{N_{s}}\end{array}\right], \ldots . .(9) \quad \boldsymbol{B}=\left[\begin{array}{c}\widetilde{\boldsymbol{b}}_{1} \\ \widetilde{\boldsymbol{b}}_{2} \\ \vdots \\ \tilde{\boldsymbol{b}}_{N_{s}}\end{array}\right] \ldots \ldots(10)$

Equation (6) for one period is now expressed in the form

$A(\boldsymbol{X})=\boldsymbol{B}$

To accelerate convergence to the steady state, $\boldsymbol{X}$ is decomposed into fast and slowly converging components as follows:

$$
\boldsymbol{X}=\tilde{\boldsymbol{X}}+\mathrm{Wp}
$$

where $\boldsymbol{p} \in \mathbb{R}^{M N}$ and $\mathrm{W} \in \mathbb{R}^{M N \mathrm{~s} \times M N}$ represent the correction vector and matrix composed of the slowly converging components whose explicit forms will be described below. The constant $M$ represents the degree of the correction. The approximated solution $\boldsymbol{X}$ given by (12) is substituted to (11) to obtain the residual vector given by

$$
\boldsymbol{r}^{\prime}=\boldsymbol{B}-\boldsymbol{A}(\tilde{\boldsymbol{X}}+\mathrm{W} \boldsymbol{p})
$$

We expand (13) around $\tilde{\boldsymbol{X}}$ for linearization, and then employ the Galerkin approximation that $\boldsymbol{r}^{\prime}$ in (13) is orthogonal to the column vectors in $\mathrm{W}$ to obtain the correction equation given by

$$
\mathrm{W}^{\mathrm{t}} \mathrm{AWp}=\mathrm{W}^{\mathrm{t}} \boldsymbol{r}
$$

where $\boldsymbol{r}=\boldsymbol{B}-\boldsymbol{A}(\tilde{\boldsymbol{X}})$ and

$$
\begin{aligned}
\mathrm{A} & =\left[\begin{array}{cccccc}
\mathrm{C}_{1} & 0 & 0 & \cdots & 0 & \mathrm{P}_{N_{S}} \\
\mathrm{P}_{1} & \mathrm{C}_{2} & 0 & \cdots & 0 & 0 \\
0 & \ddots & \mathrm{P}_{n-1} & \mathrm{C}_{n} & \ddots & 0 \\
\vdots & & & \\
0 & 0 & 0 & \cdots & \mathrm{P}_{N_{S}-1} & \mathrm{C}_{N_{S}}
\end{array}\right] . \\
\mathrm{C}_{n} & =\frac{\partial \boldsymbol{C}\left(\boldsymbol{x}_{n}\right)}{\partial \boldsymbol{x}_{n}}=\theta \frac{\partial \boldsymbol{f}\left(\boldsymbol{x}_{n}\right)}{\partial \boldsymbol{x}_{n}}+\frac{\mathrm{C}}{\Delta t} . \\
\mathrm{P}_{n} & =\frac{\partial \boldsymbol{P}\left(\boldsymbol{x}_{n}\right)}{\partial \boldsymbol{x}_{n}}=(1-\theta) \frac{\partial \boldsymbol{f}\left(\boldsymbol{x}_{n}\right)}{\boldsymbol{x}_{n}}-\frac{\mathrm{C}}{\Delta t} .
\end{aligned}
$$

The correction matrix $\mathrm{W} \in \mathbb{R}^{N N \mathrm{~s} \times N}$ of the 0 th order $(M=1)$, in which the slowly converging components are assumed to be temporally constant, is given by

$$
\mathrm{W}=\left[\begin{array}{llll}
\mathrm{I} & \mathrm{I} & \cdots & \mathrm{I}
\end{array}\right]^{\mathrm{t}}
$$

where $I \in \mathbb{R}^{N \times N}$ is the unit matrix. By substituting (17) to the 
left-hand side of (14), one has

$$
\mathrm{W}^{\mathrm{t}} \mathrm{AW}=\sum_{n=1}^{N_{S}}\left(\mathrm{C}_{n}+\mathrm{P}_{n}\right)
$$

Then it is assumed that (11) can be solved with sufficiently high accuracy except at $n=0$ where imperfect periodicity would result in non-negligible residuals. Under this assumption, the residual can be expressed in the form

$$
\boldsymbol{r}=\left[\begin{array}{c}
\boldsymbol{P}\left(\boldsymbol{x}_{N_{S}}\right)-\boldsymbol{P}\left(\boldsymbol{x}_{0}\right) \\
\mathbf{0} \\
\vdots \\
\mathbf{0}
\end{array}\right] .
$$

Substitution of (18) and (19) to (14) yields

$$
\left[\sum_{n=1}^{N_{S}}\left(\mathrm{C}_{n}+\mathrm{P}_{n}\right)\right] \boldsymbol{p}=\boldsymbol{P}\left(\boldsymbol{x}_{N_{S}}\right)-\boldsymbol{P}\left(\boldsymbol{x}_{0}\right) .
$$

By solving (20) for $\boldsymbol{p}, \boldsymbol{X}$ is corrected from (12).

Convergence to the steady state can further be accelerated by use of the correction of the 1 st order $(M=2)$ in which the slowly converging components are assumed to be temporally constant and linear. The corresponding correction matrix is given by

$$
\mathrm{W}=\left[\begin{array}{ccccc}
\mathrm{I} & \cdots & \mathrm{I} & \cdots & \mathrm{I} \\
-\mathrm{I} & \cdots & f_{n} \mathrm{I} & \cdots & \mathrm{I}
\end{array}\right]^{\mathrm{t}}
$$

where

$f_{n}=\frac{2 n-N_{S}}{N_{S}}, \quad\left(0 \leq n \leq N_{S}\right)$.

By substituting (21) to (14), one obtains

$$
\begin{aligned}
& {\left[\begin{array}{ll}
\mathrm{A}_{11} & \mathrm{~A}_{12} \\
\mathrm{~A}_{21} & \mathrm{~A}_{22}
\end{array}\right]\left[\begin{array}{l}
\boldsymbol{p}_{0} \\
\boldsymbol{p}_{1}
\end{array}\right]=\left[\begin{array}{c}
\boldsymbol{r}_{1} \\
-\boldsymbol{r}_{1}
\end{array}\right]} \\
& \mathrm{A}_{11}=\sum_{n=1}^{N_{S}}\left(\mathrm{C}_{n}+\mathrm{P}_{n}\right) \\
& \mathrm{A}_{12}=\sum_{n=1}^{N_{S}} f_{n}\left(\mathrm{C}_{n}+\mathrm{P}_{n}\right) \\
& \mathrm{A}_{21}=\sum_{n=1}^{N_{S}} f_{n} \mathrm{C}_{n}+\sum_{m=1}^{N_{S}-1} f_{m-1} \mathrm{P}_{m}+f_{0} \mathrm{P}_{N_{S}} \\
& \mathrm{~A}_{22}=\sum_{n=1}^{N_{S}} f_{n}^{2} \mathrm{C}_{n}+\sum_{m=1}^{N_{S}-1} f_{m} f_{m-1} \mathrm{P}_{m}+f_{0} f_{N_{S}} \mathrm{P}_{N_{S}} .
\end{aligned}
$$

The unknown $\boldsymbol{x}_{n}$ is then corrected with the vectors $\boldsymbol{p}_{0}$ and $\boldsymbol{p}_{1}$ determined by solving (22) as follows: $\boldsymbol{x}_{n}=\tilde{\boldsymbol{x}}_{n}+\boldsymbol{p}_{0}+f_{n} \boldsymbol{p}_{1}$

Although increase in the degree of the correction, e. g. from 0th to 1 st, is expected to give better convergence, it also results in increase in the unknowns in the correction equation.

\section{Effect of TP-EEC Method}

The mathematical property of the TP-EEC method has been discussed for the scalar linear diffusion equation [5]. We give here more general discussion on this method. By substituting $r$ and (14) to (12), we obtain

$$
\boldsymbol{X}=\tilde{\boldsymbol{X}}+\mathrm{W}\left(\mathrm{W}^{\mathrm{t}} \mathrm{AW}\right)^{-1} \mathrm{~W}^{\mathrm{t}}(\boldsymbol{B}-\mathrm{A} \tilde{\boldsymbol{X}}) .
$$

The steady solution to (11) is here expressed by $\boldsymbol{X}^{*}$. Then, the error $\widetilde{\boldsymbol{E}}=\boldsymbol{X}^{*}-\tilde{\boldsymbol{X}}$ is modified after the error correction in the form

$\boldsymbol{E} \approx \mathrm{P} \widetilde{\boldsymbol{E}}$

where $\boldsymbol{E}=\boldsymbol{X}^{*}-\boldsymbol{X}$ and $\mathrm{P}$ is defined by

$P=I-W\left(W^{t} A W\right)^{-1} W^{t} A$

which is a projection matrix satisfying $\mathrm{P}=\mathrm{P}^{2}$. It can easily be seen from (26) that $\mathrm{PW}=0$. The error $\widetilde{\boldsymbol{E}}$ can be decomposed in the form

$\widetilde{\boldsymbol{E}}=\boldsymbol{E}_{\text {slow }}+\boldsymbol{E}_{\text {fast }}$

where

$\boldsymbol{E}_{\text {slow }} \in \operatorname{Range}(\mathrm{W}), \quad \boldsymbol{E}_{\text {fast }} \in \operatorname{Ker}\left(\mathrm{W}^{\mathrm{t}} \mathrm{A}\right)$.

Therein, the errors $\boldsymbol{E}_{\text {slow }}$ and $\boldsymbol{E}_{\text {fast }}$ represent the slowly and fast converging errors, and they satisfy A-orthogonal relation $\left(\boldsymbol{E}_{\text {slow }}\right.$, $\left.\boldsymbol{E}_{\text {fast }}\right)_{\mathrm{A}}=0$. It can be seen from (25) to (28) that the slowly converging error is eliminated as $\mathrm{P} \boldsymbol{E}_{\text {slow }}=0$ by the TP-EEC method. On the other hand, $\boldsymbol{E}_{\text {fast }}$ has no effects from the correction because of the property $\mathrm{P} \boldsymbol{E}_{\text {fast }}=\boldsymbol{E}_{\text {fast }}$. Thus this error component is reduced by the iterative solution of (11) where its convergence is expected to be fast by definition. It is known that the multigrid method, which effectively eliminates the slowly converging components with spatially smooth profiles by mapping them to coarser meshes, is also based on the above mentioned decomposition and selective elimination [14]. More detailed discussions on the TP-EEC method are can be found in [15]. 
IV. NUMERICAL RESULT

\section{A. $\quad$ CR Diode Series Circuit}

The half-wave dipole antenna loaded by the non-linear circuit shown in Fig. 4 is analyzed by hybridization of the FDTD method and MNA to test acceleration of convergence to the steady state by applying the TP-EEC method to MNA. The nodal voltages of the non-linear circuit are obtained from MNA. The half-wave dipole antenna is assumed to be illuminated by the plane wave. The amplitude and frequency of incident wave is assumed to be $20 \mathrm{~V} / \mathrm{m}$ and $1 \mathrm{GHz}$. The size of the FDTD cell, $\Delta \mathrm{X}=\Delta \mathrm{Y}=\Delta \mathrm{Z}$, is set to $3 \mathrm{~mm}$. The perfect matched layer is employed to enforce the free space conditions on the domain boundary. The half-wave dipole antenna is parallel to $z$-axis and set to perfect conductor $\left(E_{\mathrm{z}}=0 \mathrm{~V} / \mathrm{m}\right)$.

The nonlinear circuit is composed of a capacitance, diode and resistance as shown in Fig. 5. The capacitance $C$ is set to $10 \mathrm{pF}$ and the resistance $R$ is set to $1 \mathrm{k} \Omega$. The diode circuit is assumed to obey V-I characteristics given by

$i_{\mathrm{d}}=\left\{\begin{array}{cc}1.0 \times 10^{-8} v_{\mathrm{d}} & v_{\mathrm{d}} \leq 0 \\ 2.9 \times 10^{-7}\left[\exp \left(15 v_{\mathrm{d}}\right)-1\right] & v_{\mathrm{d}} \geq 0\end{array}\right.$

where $v_{\mathrm{d}}$ is the voltage at diode. For negative voltages, the diode current is nearly zero. The explicit form of the circuit equation (5) for the circuit shown in Fig. 5 is given in the Appendix. In this analysis, $\theta$ is set to 1 in MNA and $N_{\mathrm{S}}$ is set to 200 .

Fig. 6 shows the time evolution in the voltage $V_{1}$. It can be seen in Fig. 6 that the convergence to the steady state is clearly accelerated by the present method.

Fig. 7 shows the absolute error $E$ between the steady and transient solutions. The numbers of time steps required to satisfy $|E|<0.01$ for no correction, for 0th correction, and for $1 \mathrm{st}$ order correction, are approximately 205000 steps (1025 ns), 2400 steps (12 ns) and 1600 steps (8 ns), respectively. This

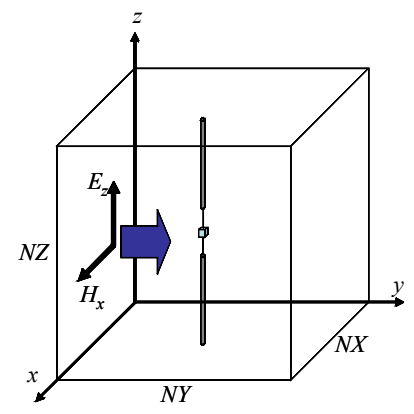

Fig. 4. Analysis model.

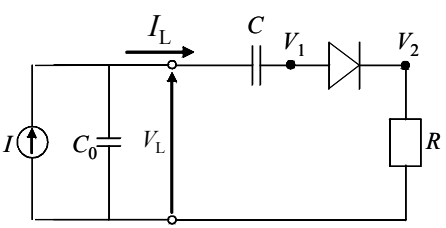

Fig. 5. CR diode serial circuit.

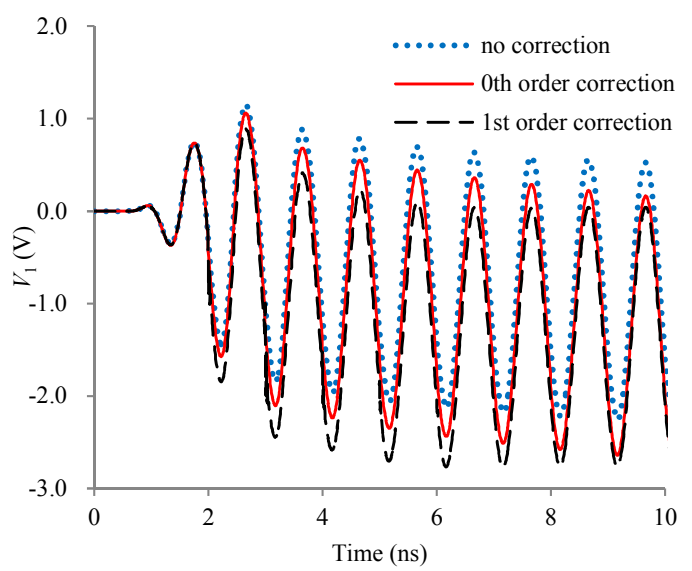

Fig. 6. Time evolution of $V_{1}$.

means that the TP-EEC method of the 1 st order correction provides convergence to the steady state 128.1 times faster than that for non-corrected computation. Moreover, the three solutions in the steady state are found to be in good agreement.

\section{B. Cockcroft-Walton Circuit}

The TP-EEC method is now applied to the half-wave dipole antenna loaded by the $\mathrm{CW}$ circuit shown in Fig. 8. The explicit form of the circuit equation (5) for the circuit shown in Fig. 8 is given in the Appendix. The parameters of the FDTD method and MNA are the same as those used in subsection 4.1.

Fig. 9 shows the time evolution of the output voltage $V_{\text {out }}$ of the $\mathrm{CW}$ circuit. It can be seen in Fig. 9 that the convergence to the steady state is clearly accelerated by the TP-EEC method. Fig. 10 shows the absolute error $E$ between the steady and transient solutions. The number of time steps required to satisfy $|E|<0.01$ for no correction, for 0th order correction, and for $1 \mathrm{st}$

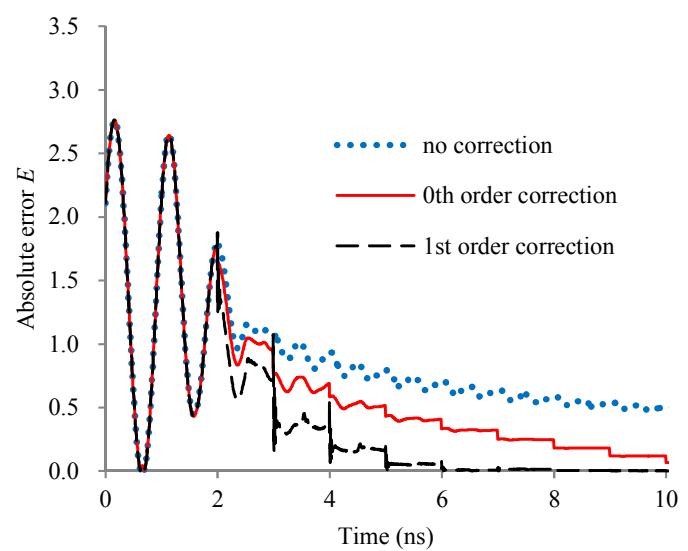

Fig. 7. Time evolution error of node voltage.

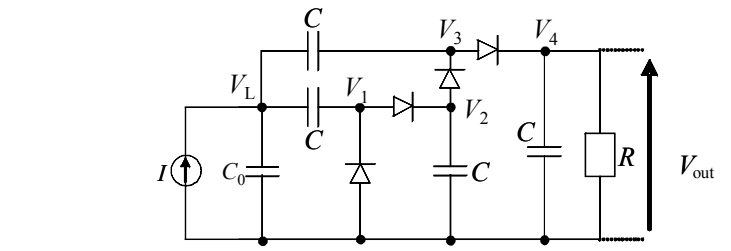

Fig. 8. Cockcroft-Walton circuit. 


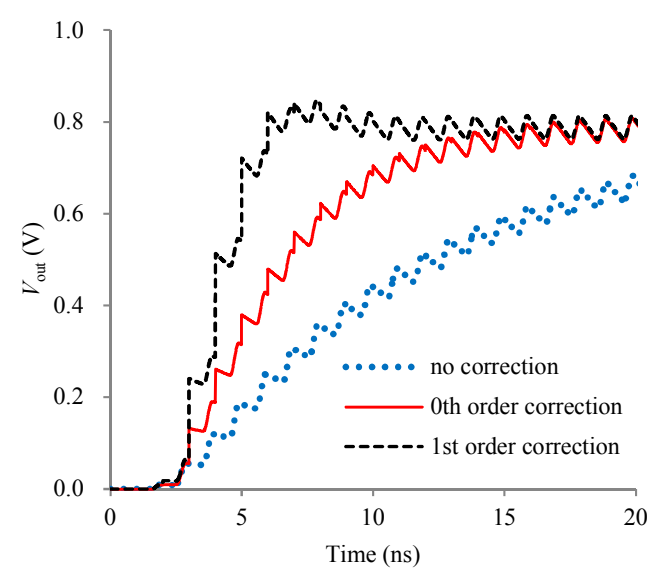

Fig. 9. Time evolution of output voltage $V_{\text {out }}$.

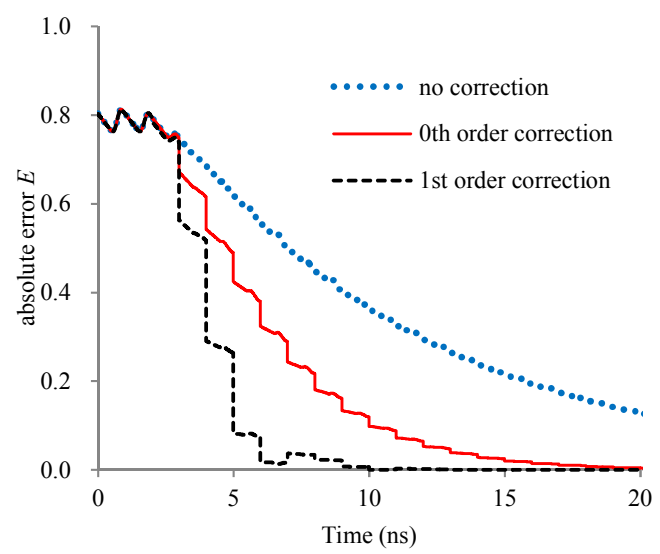

Fig. 10. Time evolution error of node voltage.

order correction, are approximately 8880 steps (44 ns), 3545 steps (18 ns) and 1800 steps (9 ns), respectively. That means that the TP-EEC method of the 1st order correction provides convergence to the steady state 4.9 times faster than that for non-corrected computation. Moreover, it is observed that the three solutions in the steady state are in good agreement. It can be seen in Fig. 7 and Fig. 10 that the effects in acceleration by TP-EEC method depend on circuits.

\section{CONCLUSIONS}

In this paper, it has been shown that convergence to the steady state of a non-linear circuit driven by antenna voltage, which was analyzed by FDTD and MNA, is effectively accelerated by using the present method. The theoretical reason why the present method can improve convergence to the steady state has been discussed. To test the present method, it has been applied to analysis of a half-wave dipole antenna loaded by non-linear circuits including diodes. It has been numerically shown that the TP-EEC method effectively accelerates convergence to the steady state.

\section{APPENDIX}

This appendix describes the nodal equations for the circuit discussed in this paper. The nodal equations for the CR diode series circuit shown in Fig. 5 are given by

$$
\begin{aligned}
& -C_{0} \frac{\mathrm{d} V_{\mathrm{L}}}{\mathrm{d} t}+C \frac{\mathrm{d}}{\mathrm{d} t}\left(V_{1}-V_{\mathrm{L}}\right)=I \\
& C \frac{\mathrm{d}}{\mathrm{d} t}\left(V_{\mathrm{L}}-V_{1}\right)-i_{\mathrm{d}}\left(V_{2}-V_{1}\right)=0 \\
& i_{\mathrm{d}}\left(V_{1}-V_{2}\right)-\frac{V_{2}}{R}=0,
\end{aligned}
$$

where $I$ is the input current obtained by the FDTD computation and $C_{0}$ is the capacitance for the FDTD cell.

The nodal equations for the $\mathrm{CW}$ circuit shown in Fig. 8 are given by

$$
\begin{aligned}
& -C_{0} \frac{\mathrm{d} V_{\mathrm{L}}}{\mathrm{d} t}+C \frac{\mathrm{d}}{\mathrm{d} t}\left(V_{1}-V_{\mathrm{L}}\right)+C \frac{\mathrm{d}}{\mathrm{d} t}\left(V_{3}-V_{\mathrm{L}}\right)=I \\
& C \frac{\mathrm{d}}{\mathrm{d} t}\left(V_{\mathrm{L}}-V_{1}\right)+i_{\mathrm{d}}\left(-V_{1}\right)-i_{\mathrm{d}}\left(V_{1}-V_{2}\right)=0 \\
& C \frac{\mathrm{d} V_{2}}{\mathrm{~d} t}+i_{\mathrm{d}}\left(V_{1}-V_{2}\right)-i_{\mathrm{d}}\left(V_{2}-V_{3}\right)=0 \\
& C \frac{\mathrm{d}}{\mathrm{d} t}\left(V_{0}-V_{3}\right)+i_{\mathrm{d}}\left(V_{2}-V_{3}\right)-i_{\mathrm{d}}\left(V_{3}-V_{4}\right)=0 \\
& -C \frac{\mathrm{d} V_{4}}{\mathrm{~d} t}+i_{\mathrm{d}}\left(V_{3}-V_{4}\right)-\frac{V_{4}}{R}=0 .
\end{aligned}
$$

\section{REFERENCES}

[1] K. S. Yee, "Numerical solution of initial boundary value problems involving Maxwell's equations in isotropic media," IEEE Trans. Antennas. Propagat, vol. AP-14, pp. 302-307, 1966

[2] A. Taflove, Computational Electrodynamics, The Finite-Difference Time-Domain Method, Artech house publishers, 1998.

[3] R. F. Harrington, Field computation by moment methods, Wiley-IEEE Press, 1993

[4] C. R. Paul, Introduction to Electromagnetic Compatibility, Wiley, 2006.

[5] Y. Takahashi, T. Tokumasu, M. Fujita, S. Wakao, T. Iwashita, M. Kanazawa, "Improvement of convergence characteristic in nonlinear transient eddy-current analyses using the error correction of time integration based on the time-periodic FEM and the EEC method," IEEJ Trans. PE, vol. 129, no. 6, pp. 791-798, 2009.

[6] Y. Takahashi, T. Tokumasu, A. Kameari, H. Kaimori, M. Fujita, T. Iwashita and S. Wakao, "Convergence Acceleration of Time-Periodic Electromagnetic Field Analysis by the Singularity Decomposition-Explicit Error Correction Method," IEEE Trans. Magn., vol. 46, no. 8, pp. 2947-2950, Aug. 2010.

[7] T. Iwashita, T. Mifune, and M. Shimasaki, "Similarities between implicit correction multigrid method and A-phi formulation in electromagnetic field analysis," IEEE Trans. Magn., vol. 44, no. 6, pp. 946-949, 2008.

[8] H. Igarashi, and K. Watanabe, "Deflation Techniques for computational electromagnetism: Theoretical considerations, IEEE Trans. Magn., vol. 47. No. 5, pp. 1438-1441, 2011.

[9] C. Vuik, A. Segal, and J. A. Meijerink, “An efficient preconditioned CG method for the solution of a class of layered problems with extreme contrasts in the coefficients," J. Comp. Phys., vol. 152, pp. 385-403, 1999.

[10] Y. Saad, M. Yeung, J. Erhel, and F. Guyomarch, "A deflated version of the conjugate gradient algorithm," SIAM J. Sci. Comput., vol. 21, no. 5, pp. 1909-1926, 2000.

[11] Y. Watanabe, K. Watanabe, H. Igarashi, "Optimization of meander line antenna considering coupling between non-linear circuit and 
electromagnetic waves for UHF-band RFID," IEEE Trans. Mag.,vol. 47, no. 5, pp. 1506-1509, May, 2011.

[12] V. A. Thomas, M. E. Jones, M. Piket-May. A. Taflove, and E. Harrigan, "The use of SPICE lumped circuits as sub-grid models for FDTD analysis," IEEE Micr. Guid. Wave Lett., vol. 4, no. 5, pp. 141-143, 1994

[13] M. J. Piket-May, A. Taflove, and J. Baron, "FDTD modeling of digital signal propagation in 3-D circuits with passive and active loads," IEEE Trans. Microwave Theory \& Techniques, vol. 42, pp. 1514-1523, Aug. 1994.

[14] D. Braess, and W. Hackbusch, "A new convergence proof for the multigrid method including the V-cycle," SIAM J. Numer. Anal. Vol. 20 no. 5, pp. 967-975, 1983.

[15] H. Igarashi, Y. Watanabe, Y. Itoh, K. Watanabe, "On error correction methods for acceleration of convergence," submitted to IEEE Trans. Magn.

Yuta Watanabe (M'10) received B.S. and M.I. degrees in engineering from Akita National College of Technology and Hokkaido University, Japan, in 2008 and 2010, respectively. He has been a student of the doctoral course at the Graduate School of Information Science and Technology [of] at Hokkaido University since 2010. His primary research interest is in the area of computational electromagnetics and design optimization.

Hajime Igarashi (M'95) received B.E. and M.E. degrees in electrical engineering from Hokkaido University, Japan, in 1982 and 1984, respectively, and a Ph.D. degree in engineering from Hokkaido University in 1992 . He worked as a research engineer at Canon Co. Ltd., [during] from 1984-1989. [During] From 1989-1999, he was a research associate at the Faculty of Engineering [in] at Hokkaido University. He was a guest researcher at Berlin Technical University, Germany, under support from the Humboldt Foundation [during] from 1995-1997. He was an associate professor [during] from 1999-2004 at Kagawa University, Japan, and Hokkaido University, and has been a professor at the Graduate School of Information Science and Technology [of] at Hokkaido University since 2004. His research area is computational electromagnetism, design optimization and RFID technologies. He has authored and coauthored more than 90 peer-reviewed journal papers. 